\title{
Behaviour of the periods in double mode RR Lyrae, V26 in M15
}

\author{
M. Paparó ${ }^{1}$, B. Szeidl ${ }^{1}$, S. M. Saad ${ }^{2}$, Z. Kolláth ${ }^{1}$, and M. S. Abu Elazm ${ }^{2}$ \\ 1 Konkoly Observatory, 1525 Budapest XII, PO Box 67, Hungary \\ e-mail: paparo@konkoly.hu, szeidl@konkoly.hu, kollath@konkoly.hu \\ ${ }^{2}$ Helwan Institute of Astronomy and Geophysics, Cairo, Egypt \\ e-mail: astro3@frcu.eun.eg
}

Received 3 September 2001/ Accepted 30 April 2002

\begin{abstract}
The changes in the periods of the fundamental and first overtone pulsation of the variable V26 in the globular cluster M15 are studied. The analysis was based on published observations. The method of investigation was outlined in our previous paper (Paparó et al. 1998). The Fourier phase diagrams for both frequencies have been constructed. General guidelines concerning the cycle counting problems are discussed. In both periods a sudden increase took place around JD $2431500(\approx 1945)$ and the changes were the same in sign but slightly different in magnitude. The period change behaviour of the fundamental mode was noticed for the first time. Before the abrupt change the fundamental and first overtone periods were $P_{0}=0^{\mathrm{d}} 538898$ and $P_{1}=0.402256$ days and after it $P_{0}=0.538979$ and $P_{1}=0.402273$ days. Although the fundamental component is very weak, there is a remarkable concordance between our period after the abrupt change and the period derived by Clement \& Walker (1991). The disagreement between the observed period changes and the expectation of stellar evolutionary theory strongly suggest that period changes are dominated by short term changes governed by the physics of the star. Fast changes in oscillation frequency such as in V26/M15 cannot take place without sudden changes in the stellar structure. The possible physical processes are the mixing events in a small part of the semiconvective zone (Sweigart \& Renzini 1979) or the dredge up of a small amount of helium by occasional convection overshooting (Cox 1998).
\end{abstract}

Key words. stars: individual: V26 in M15 - stars: oscillations - stars: variables: RR Lyrae - globular cluster: individual: M15

\section{Introduction}

The study of period changes of RR Lyrae stars in globular clusters is considered to be a powerful test of the evolutionary theories of horizontal branch stars. As stellar evolution results in slow changes in the star's physical parameters (e.g. in stellar radius), one may expect that the accumulation of subtle changes in the periods might reveal the trend and the size of the period changes.

On the one hand the period changes observed often differ in sign and in rate from the theoretically predicted values, and on the other hand the complex structure of the O-C diagram of some RR Lyrae stars suggests that some kind of "noise" may be superimposed on the secular variations of the periods. Abrupt changes in the periods are also characteristic of some of the stars (Szeidl 1975; Smith \& Sandage 1981). The source of "noise" and the abrupt changes have been attributed to discrete mixing events in the semiconvective zones (Sweigart \& Renzini 1979) or they may have a hydromagnetic origin (Stothers 1980).

Theoretical and evolutionary consideration suggested that the relative changes in the period of the fundamental and first overtone of an $\mathrm{RR}_{\mathrm{d}} \operatorname{star}\left(1 / P_{0} * \mathrm{~d} P_{0} / \mathrm{d} t\right.$ and $\left.1 / P_{1} * \mathrm{~d} P_{1} / \mathrm{d} t\right)$

Send offprint requests to: M. Paparó, e-mail: paparo@konkoly.hu should be of the same sign and about the same rate. Paparó et al. (1998) (Paper I) have demonstrated that no strict correlation exists between the changes in the periods of the two pulsational modes of an $R_{d}$ star, neither in rate nor in sign. Thus, these investigations questioned the simple idea that the linear period changes reflect the evolution of RR Lyrae stars on the horizontal branch (HB). All the stars studied in Paper I, have shown slow linear changes in their dominant (first overtone) frequency (except V54, which showed a small period jump as well). V26 was not included in the previous investigation because its first overtone frequency had a large abrupt change around 1945 or JD 2431500 (Silbermann \& Smith 1995a) and its preliminary study (Paparó et al. 1997) also indicated that the frequency of the fundamental mode was subject to a sudden change around the same time. In view of this, V26 deserves special attention.

\section{Data}

The investigation of RR Lyrae stars in M15 was started by Bailey (1919) in 1896. The definition of variable stars in M15 as variable $(V$ or $v$ ) and the running number of Bailey's list (26 in our case) has been accepted in all catalogues (e.g. Sawyer Hogg 1973). In the ensuing nine decades a great number of photographic observations have been obtained by 
Table 1. Distribution of observations used in the present work.

\begin{tabular}{|c|c|c|c|}
\hline Year & $\begin{array}{l}\text { Number of ob } \\
\text { in } p g / B \text { band }\end{array}$ & $\begin{array}{l}\text { servations } \\
\text { in } V \text { band }\end{array}$ & Source \\
\hline 1915 & $35 \mathrm{pg}$ & & Bailey (1919) \\
\hline 1916 & $27 \mathrm{pg}$ & & $"$ \\
\hline 1925 & $33 \mathrm{pg}$ & & Fritze (1963) \\
\hline 1933 & $13 \mathrm{pg}$ & & " \\
\hline 1937 & $29 \mathrm{pg}$ & & Barlai (1989) \\
\hline 1938 & $86 \mathrm{pg}$ & & $"$ \\
\hline 1939 & $14 \mathrm{pg}$ & & ” \\
\hline 1940 & $11 \mathrm{pg}$ & & ” \\
\hline 1941 & $18 \mathrm{pg}$ & & 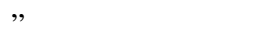 \\
\hline 1950 & $5 \mathrm{pg}$ & & $"$ \\
\hline 1951 & $80 \mathrm{pg}$ & & ” \\
\hline 1952 & $15 \mathrm{pg}$ & & " \\
\hline 1953 & $5 \mathrm{pg}$ & & $"$ \\
\hline 1954 & $154 \mathrm{pg}$ & & Grubissich (1956) \\
\hline \multirow[t]{2}{*}{1955} & $34 \mathrm{pg}$ & & $”$ \\
\hline & $1 \mathrm{pg}$ & & Barlai (1989) \\
\hline 1956 & $16 \mathrm{pg}$ & & $"$ \\
\hline \multirow[t]{2}{*}{1957} & $25 \mathrm{pg}$ & & $"$ \\
\hline & $3 \mathrm{~B}$ & $6 \mathrm{~V}$ & Sandage et al. (1981) \\
\hline 1958 & $42 \mathrm{~B}$ & $42 \mathrm{~V}$ & $"$ \\
\hline 1959 & $17 \mathrm{~B}$ & $16 \mathrm{~V}$ & $"$ \\
\hline 1963 & $25 \mathrm{pg}$ & & Barlai (1989) \\
\hline \multirow[t]{2}{*}{1966} & $15 \mathrm{pg}$ & & $"$ \\
\hline & $7 \mathrm{~B}$ & & Wesselink (1974) \\
\hline 1967 & $20 \mathrm{~B}$ & & $"$ \\
\hline 1968 & $19 \mathrm{~B}$ & & $"$ \\
\hline 1973 & & $1 \mathrm{~V}$ & Bingham et al. (1984) \\
\hline 1974 & $9 \mathrm{~B}$ & $12 \mathrm{~V}$ & $"$ \\
\hline 1975 & $4 \mathrm{~B}$ & $2 \mathrm{~V}$ & $"$ \\
\hline 1976 & & $31 \mathrm{~V}$ & Filippenko \& Simon (1981) \\
\hline \multirow[t]{2}{*}{1977} & & $60 \mathrm{~V}$ & ” \\
\hline & $9 \mathrm{~B}$ & $8 \mathrm{~V}$ & Bingham et al. (1984) \\
\hline \multirow[t]{2}{*}{1978} & & $107 \mathrm{~V}$ & Filippenko \& Simon (1981) \\
\hline & $8 \mathrm{~B}$ & $16 \mathrm{~V}$ & Bingham et al. (1984) \\
\hline 1979 & $14 \mathrm{~B}$ & $14 \mathrm{~V}$ & $"$ \\
\hline
\end{tabular}

different observers. Between 1896 and 1908 Bailey's observations were rather sporadic and scarce. Since 1915, however, there have been very many photographic observations and they are relatively uniform. We have made extensive use of the observation of Bailey (1919), Barlai (1989), Bingham et al. (1984), Filippenko \& Simon (1981), Fritze (1963), Grubissich (1956), Sandage et al. (1981) and Wesselink (1974). The observations of Makarova \& Akimova (1965) were left out of consideration because these observations were given in an unusual photometric system; Gordenko et al. (1984) did not publish individual observations for the stars they studied.

Recently we have seen the beginning of a new era in observational techniques: Silbermann \& Smith (1995b) started CCD observations of the variables in M15 in the early 1990s. Unfortunately no CCD photometric data on V26 have been obtained since the star was out of the CCD frame (Silbermann 2001).

Table 1 summarizes the number and photometric band of observations of the different years discussed in this paper.

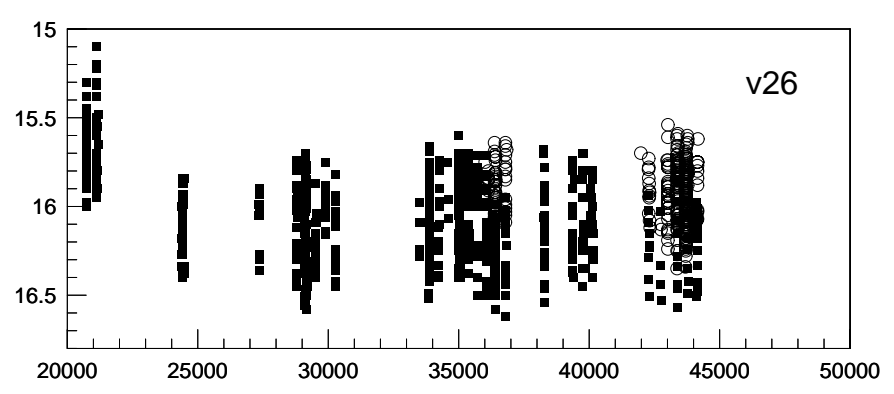

Fig. 1. Distribution of data. Squares indicate photographic (or B) observations, open circles $V$ observations. The ordinate scale is given in magnitude and the abcissa is time in days JD -2400000 .

The distribution of data used are shown in Fig. 1 (filled squares: $p g / B$ observations, open circles: $V$ observations). The separation into segments is given later in Table 4.

Over the past 90 years observations have been carried out by a variety of techniques, telescopes of different size and focal ratio, etc., which may have serious effects on the photometry. Generally speaking reduction of the data to the same photometric system is not an easy task albeit the magnitudes of comparison stars used by different observers are known. However, in our case the magnitude scale of the different photometric systems are insignificant: only the zero points show slight differences. The photographic light curves of most observers are well-matched, so all the $m_{p g}$ and $B$ data are treated together and used as a coherent data set. The zero point in each case is treated as a free parameter in our investigation.

As we see in Fig. 1 the mean values from the different observational tracks are the same except for the observations of Bailey which are brighter by about $0.6 \mathrm{mag}$. We may say as an explanation that the photographic technique was in its early stages compared with the later generally-accepted sensitivity range of emulsion. However, in this case all the other stars in M15 measured by Bailey on the same set of plates should show a similar difference in the mean value compared with his other observations. As is shown by the distribution of data of other double mode RR Lyrae stars (Fig. 1 in Paper I) there is not such a large difference in the mean light level for the other stars. Bailey (1919) used the same set of standard stars for all variables except for V28. The Hungarian photographic plates were carefully checked for possible contamination by a faint star near to V26 but contamination was ruled out as an explanation. V26 has no specific position in the cluster. It is not near the center of the cluster which would make questionable the precise photometry. At present, it would seem that there is no acceptable explanation for Bailey's observation for the mean light level of V26.

We accepted the mean error of the observations as $0.15 \mathrm{mag}$ throughout our analysis, which is characteristic of the photographic observations, but overestimates the error of the highquality data of Sandage et al. (1981) and Bingham et al. (1984).

\section{Analysis}

The available observational data do not readily lend themselves to an analysis of the changes in the frequencies of 
Table 2. Period derived by different authors.

\begin{tabular}{|c|c|c|c|}
\hline $\begin{array}{l}P_{1} \\
\text { days }\end{array}$ & $\begin{array}{l}P_{0} \\
\text { days }\end{array}$ & Source & Remark \\
\hline 0.402326 & & Bailey (1919) & \\
\hline 0.402270 & & Grubissich (1956) & \\
\hline 0.4023068 & & Fritze (1962) & \\
\hline 0.402278 & & Wesselink (1974) & \\
\hline 0.402270 & & Smith \& Sandage (1981) & \\
\hline 0.4023 & $\begin{array}{l}0.5430 \\
0.5398\end{array}$ & $\begin{array}{l}\text { Hodson \& Cox (1982) } \\
\text { " }\end{array}$ & $\begin{array}{l}\text { SKS } \\
\text { FS }\end{array}$ \\
\hline 0.402275 & 0.5430 & Cox et al. (1983) & SKS \\
\hline 0.402260 & 0.53913 & Nemec (1985) & SKS \\
\hline 0.402268 & 0.53892 & Jurcsik \& Barlai (1990) & \\
\hline 0.402272 & 0.538990 & Clement \& Walker (1991) & $\mathrm{G}+\mathrm{SKS}$ \\
\hline 0.402280 & 0.53896 & " & $\mathrm{BCDF}$ \\
\hline \multicolumn{4}{|c|}{$\begin{array}{l}\text { Remarks: SKS: only Sandage et al.'s (1981) data, } \\
\text { FS: only Filippenko \& Simon's (1981) data, } \\
\text { G+SKS: only Grubissich (1956) and Sandage et al.'s (1981) data, } \\
\text { BCDF: only Bingham et al.'s (1984) data }\end{array}$} \\
\hline
\end{tabular}

both pulsational modes by the usual techniques (traditional $\mathrm{O}-\mathrm{C}$, Fourier, PDM). Therefore the method of analysis (Fourier phase method) described in detail in Paper I was used to derive the changes in the fundamental and first overtone period of V26/M15.

The main point of the method is that by fixing the frequencies and amplitudes $\left(a_{i j}\right)$ only the $\Phi_{i j}$ phases are left as free parameters in the Fourier decomposition of the light curve:

$m=a_{0}+\sum_{i j} a_{i j} \sin \left(2 \pi f_{i j}\left(t-t_{\mathrm{o}}\right)+\Phi_{i j}\right)$

where $f_{i j}$ is the linear combination of the fundamental $\left(f_{0}\right)$ and first overtone $\left(f_{1}\right)$ frequencies. The zero point is marked by $a_{0}$. The phase, $\Phi_{i j}$ is calculated from an epoch $\left(t_{0}\right)$.

In this way the reduced numbers of free parameters allowed us to use more sporadic observations as well. The method is not sensitive to small changes in the amplitude (tested in Paper I). For V26 the errors of the phase due to the errors in amplitude determination are very small: $\sigma\left(\Phi_{10} / 2 \pi\right)=3 \sigma_{a}$ and $\sigma\left(\Phi_{01} / 2 \pi\right)=0.5 \sigma_{a}$, where $\sigma_{a}$ is in magnitudes. This means that a $0.02 \mathrm{mag}$ error in $a_{0}$ results in an error of 0.06 in the phase of the fundamental mode. During the investigation the amplitudes of fundamental mode and first overtone were fixed at $a_{10}=0.034$ and $a_{01}=0.183$ mag that we obtained from a least squares fit for the whole data set. This resulted in a 1:5-1:6 amplitude ratio. The variations in the phases $\Phi_{10}$ and $\Phi_{01}$ reflect the changes in the frequencies if the fixed values of the frequencies are precise enough or the cycle counting problem can be treated appropriately. The zero point $a_{\mathrm{o}}$ was also treated as a free parameter - as permitted by the Fourier method.

It is well known that the period changes of single mode RR Lyrae stars given by the O-C diagram can be studied when the periods are precisely known. The rate of period change over 30-40 years results in changes of $10^{-6}$ days (Hoffmeister et al. 1985). We should expect that in order to investigate period changes for double mode RR Lyrae stars one needs as precise period determinations as for single mode RR Lyrae stars.

However, the possibility of obtaining as precise periods for double mode RR Lyrae stars as for single mode RR Lyrae stars is not an easy task. Even for the same length of observation, the increased number of parameters decreases the precision of the determination. The interaction of modes has only a slight effect on the larger amplitude mode whereas the effect is much stronger for the smaller amplitude modes.

There is general agreement on the first overtone period of V26/M15 derived by the authors (listed in Table 2) of up to $10^{-5}$ days. Since the total amplitude of the fundamental oscillation is much less than that of the first overtone pulsation (about a quarter of that published in the literature), the result from determining the frequency of the fundamental mode is somewhat doubtful using shorter data sets. In fact, the fundamental oscillation of V26/M15 sometimes became insignificant (e.g. in 1938) and the star did not show a measurable double mode nature in the set of Babelsberg observations (Jurcsik \& Barlai 1990; Purdue et al. 1995). However, on analysing the observations of Sandage et al. (1981) and Filippenko \& Simon (1981), Hodson \& Cox (1982) came to the conclusion that the ratio of the amplitudes of the first overtone to fundamental mode pulsation of V26/M15 had remained unchanged within the error limits. Because of the very small fundamental component in the star's pulsation, Kovács et al. (1986) left it out of their analysis. Clement \& Walker (1991), Jurcsik \& Barlai (1990) and Nemec (1985) included the star in their thorough analysis. Their investigations led to concordant results for both the fundamental and the first overtone oscillation. The general agreement on the fundamental mode period of V26/M15 (see Table 2) is much weaker than for the first overtone. Differences are up to $10^{-3}, 10^{-4}$ or $10^{-5}$ days depending on the length of the data set involved in the analysis.

In this overview we have tried to emphasize how important the precise value of a period determination is if we are interested in a meaningful period change interpretation.

Incidentally, in connection with V26/M15 we can show the strength of the Fourier phase method in finding the precise true period and what the range of discrepancy is in the period where the method is still usable. For V26/M15 the accepted starting values of the modes were taken from the paper by Nemec (1985). The value of 0.402260 days for the first overtone proved to be an appropriate starting value since the resulting phase values immediately showed similar regular structures (see in Fig. 3). However, his less precise 0.53913 day value for the fundamental mode illustrated how the phase values suffered from the cycle counting problem.

There are two parameters that influence the appearance of the phase diagram, viz. $\Delta f=f_{\text {true }}-f_{\text {false }}$ representing the correction to get the true frequency and $\Delta T$, given the time interval that we use for obtaining a certain phase value. A specific time interval, marked by $\Delta T_{\text {limit }}$, is defined by

$\Delta f * \Delta T_{\text {limit }}= \pm 1$

where $\Delta f$ is given in cycle/day, $\Delta T_{\text {limit }}$ is given in days. Equation (2) shows that the difference between the true and 


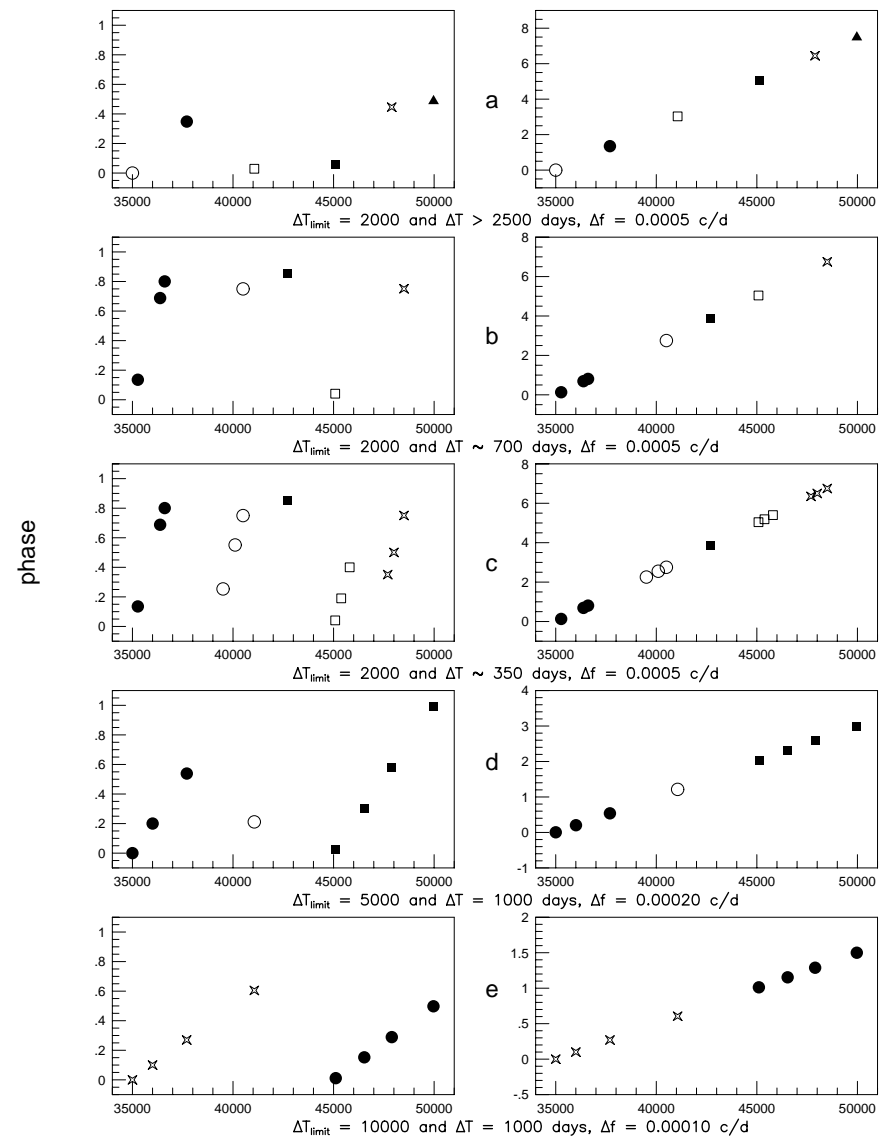

Fig. 2. Test cases for solving the cycle counting problems.

false periods accumulates and beyond the $\Delta T_{\text {limit }}$ time interval it reaches a full cycle. This means that we have to solve the serious problem of cycle counting for a gapped data set. In Fig. 2 we present some test cases calculated with different $\Delta f$ and $\Delta T$ values. The phases were calculated using the fundamental period of V26 shifted by the $\Delta f$ values. At a given $\Delta f$ value if our choice is too large, $\Delta T>\Delta T_{\text {limit }}$, then the phase values show a distribution where one or more cycles are missing between each phase point (panel $a$ ). In this case there is no way to reconstruct a continuous phase diagram. Since for the test case we knew the number of missing cycles, we reconstructed the straight line for illustration purposes. This covers a phase range of 8 cycles, thereby showing the very inaccurate value of the period used. The interpretation of such a diagram (left side), as no regular period change is apparently present, is misleading. In this case we see only the poor treatment of the cycle counting problem. Our suggestion is that $\Delta T<\Delta T_{\text {limit }}$ is applicable.

We should go further in our limitation. Our aim is to have 2 or 3 phase values between 0 and 1 without missing any cycle. These phase values will define a line with a definite slope. To get 2 or 3 phase values in the $\Delta T_{\text {limit }}$ interval we have to use $\Delta T \leq \Delta T_{\text {limit }} / 2$ or $/ 3$ time interval. In practice the $\Delta T$ time intervals do not need to be exactly the same but their sum must not exceed $\Delta T_{\text {limit }}$. Even for a single straight line, with a longer compact data set (panel $b$ ), the other phase values can be arranged along a continuous straight line increasing or decreasing the resulting phase values by $\pm 1,2$, etc. (right side panels). Obviously more than one straight line facilitates the arrangement (panel $c$ ). We should say that the lowest possible value of $\Delta T$ is desirable but the lower limit is strongly connected with the distribution of observations and the noise level. If the data are scarce there may be no way to find 2 or $3 * \Delta T$ time intervals within the $\Delta T_{\text {limit }}$. In this case there is also no way to overcome the cycle counting problem.

In the case of V26/M15 2-3 years were chosen as $\Delta T$, not equally long for each group of data. Three straight line sections were observed. However, at the beginning or at the end where observations were scarce, the $\Delta T>\Delta T_{\text {limit }}$ was used and each phase value had a missing cycle between one and the next. The three straight sections allowed us to reconstruct a continuous straight line unambiguously.

The value of the other parameter $(\Delta f)$ is not known in advance. We should consider the effect of the different $\Delta f$ at a fixed $\Delta T$. If $\Delta f$ is too large then we have the same effect as in the case of too large $\Delta T$ and the cycle counting problem is untreatable (panel $a$ ). To check whether the cycle counting problem is present, it is suggested that $\Delta T$ be decreased (as in panel $b$ ). If a smaller $\Delta T$ reproduces the straight line section then the cycle counting problem is treatable. If there are no straight lines then a new starting value for the frequency is suggested. Bearing in mind that the period of $R_{d}$ stars is precise up to $10^{-2}$ or $10^{-3}$ days, the cycle/month $(\approx 0.03 \mathrm{c} / \mathrm{d})$, cycle/year $(\approx 0.0027 \mathrm{c} / \mathrm{d})$ or cycle $/ 3$ years $(\approx 0.009 \mathrm{c} / \mathrm{d})$ aliases of the period used can serve as a new starting value (panels $d$ and $e$ ). As soon as the starting value is close to the true period ( $\Delta f$ is small enough) the straight line sections of the phase values appear and the cycle counting problem becomes treatable. The inclination of the continuous straight line gives the final correction of the starting value, i.e. the new, true period of the pulsation. If the $\Delta f$ value is sufficiently small, then regular behaviour of the phase diagram immediately appears, if it exists. We give a word of warning here: it is strongly recommended that before making any attempt to interpret $\mathrm{O}-\mathrm{C}$ or Fourier phase diagrams one should make trials to solve the cycle counting problem.

For V26/M15 no correction was applied for the first overtone but $0.00043625 \mathrm{c} / \mathrm{d}$ correction was applied for the fundamental mode starting value given by Nemec (1985). This correction was obtained from a straight line in a range of phases between \pm 6 . On repeating the phase counting procedure with the corrected new value a similar regular distribution of phases between 0 and 1 appeared for the fundamental mode that we had found for the first overtone.

As a final test the frequency spectrum was checked. After prewhitening with the first overtone and its harmonics, and linear combinations, the closely spaced peaks near to the new value of the fundamental mode were used to obtain the Fourier phase diagrams. The closely spaced peaks (cycle/many years aliases) produced a less clean Fourier phase diagram than the given $f_{0}$ value. On changing the values between the half width of the given peak some improvement occurred. The finally accepted Fourier phase diagram with the new value is presented in Fig. 3. Table 4 contains the JD dates of the first and last points in each data group used to calculate the phases. The phases and errors correspond to Fig. 3. 

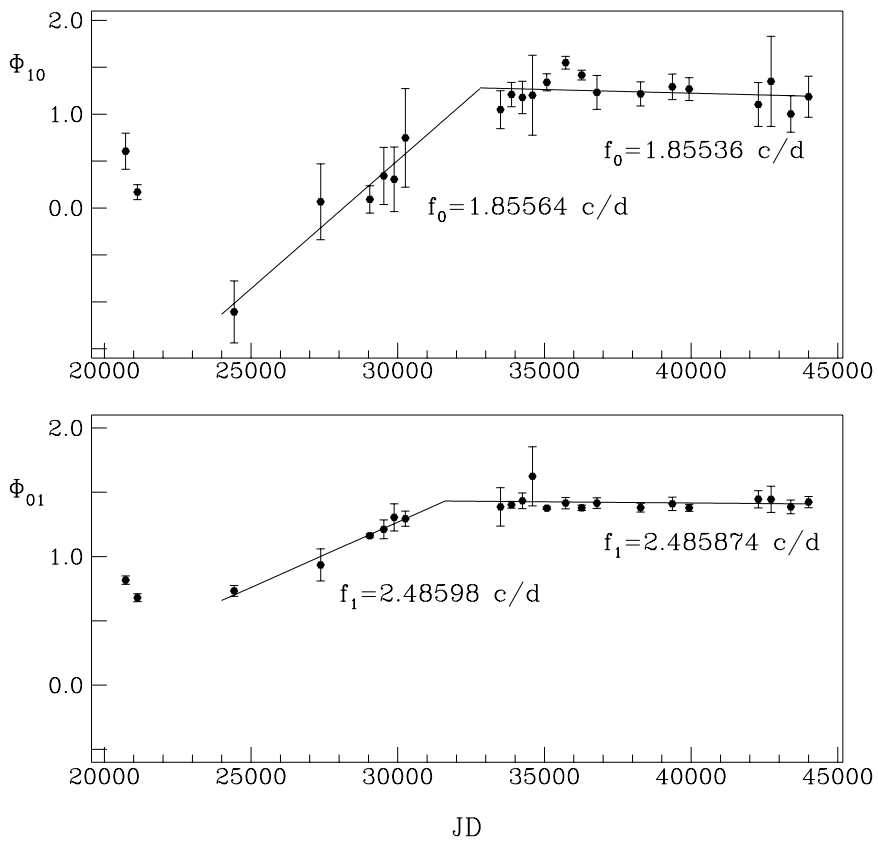

Fig. 3. Fourier phase diagram for the fundamental (upper panel) and first overtone (lower panel) modes. Possible explanation for the deviating points is given in the text.

Table 3. Frequencies of the fundamental and first overtone modes before and after the abrupt change.

\begin{tabular}{lll}
\hline \hline Frequencies & $\begin{array}{l}\text { Before } \\
\text { cycle/day }\end{array}$ & $\begin{array}{l}\text { After } \\
\text { cycle/day }\end{array}$ \\
\hline $\begin{array}{l}\text { fundamental } f_{0} \\
\text { overtone } f_{1}\end{array}$ & $\begin{array}{l}1.85564 \pm 16 \times 10^{-5} \\
2.48598 \pm 2 \times 10^{-5}\end{array}$ & $\begin{array}{l}1.855360 \pm 10 \times 10^{-6} \\
2.485874 \pm 3 \times 10^{-6}\end{array}$ \\
\hline
\end{tabular}

The deviation of Bailey's phases from the straight line is obvious. No cycle counting shift can solve the discrepancy. A possible explanation is that an abrupt period change took place at around 1914. However, this conclusion is not well-established since it is based on only two closely spaced observations.

It was known from previous studies (Silbermann \& Smith 1995a) that the $P_{1}$ primary period (first overtone period) had an abrupt change around 1945 ( $\approx$ JD 2431500), and since that time it has been more or less constant. Our present investigation shows that an abrupt change characterizes both frequencies around 1945. The exact date cannot be precisely determined because the time interval is not properly covered by observations. The period change behaviour of the fundamental mode of V26 was noticed for the first time. The large error bars before the sudden jump of the fundamental period indicate the poorer quality of the data tracks. The frequencies of both modes before and after the abrupt change are given in Table 3 . The periods obtained after the abrupt change $\left(P_{0}=0.538979\right.$ day and $P_{1}=0.402273$ day) are very close to the values derived by Clement \& Walker (1991). It is very reassuring that in such a difficult case as V26/M15 where the fundamental component is hardly noticeable, different techniques produce the same result. In Fig. 4 the folded light curves of V26 according to the
Table 4. Fourier phases and their standard deviations. $\mathrm{JD}_{1}$ and $\mathrm{JD}_{2}$ are the Julian dates of the first and last data points in each data group used to calculate the phases.

\begin{tabular}{cccccc}
\hline \hline $\mathrm{JD}_{1}$ & $\mathrm{JD}_{2}$ & $\Phi_{10} / 2 \pi$ & $\sigma\left(\Phi_{10} / 2 \pi\right)$ & $\Phi_{01} / 2 \pi$ & $\sigma\left(\Phi_{01} / 2 \pi\right)$ \\
\hline 20724.65 & 20725.99 & 0.60 & 0.10 & 0.82 & 0.02 \\
21099.72 & 21184.54 & 0.17 & 0.04 & 0.68 & 0.02 \\
24381.55 & 24474.26 & -0.11 & 0.17 & 0.73 & 0.02 \\
27334.42 & 27384.37 & 0.07 & 0.20 & 0.93 & 0.06 \\
28774.39 & 29187.32 & 0.09 & 0.07 & 1.16 & 0.01 \\
29518.31 & 29546.28 & 0.34 & 0.15 & 1.21 & 0.04 \\
29877.38 & 29879.45 & 0.31 & 0.17 & 1.30 & 0.05 \\
30259.32 & 30261.43 & 0.75 & 0.26 & 1.29 & 0.03 \\
33502.44 & 33502.53 & 1.05 & 0.10 & 1.39 & 0.07 \\
33858.43 & 33895.54 & 1.21 & 0.06 & 1.40 & 0.01 \\
34238.53 & 34270.51 & 1.18 & 0.09 & 1.43 & 0.03 \\
34573.46 & 34606.59 & 1.20 & 0.31 & 1.63 & 0.11 \\
34973.51 & 35407.28 & 1.34 & 0.04 & 1.38 & 0.01 \\
35720.32 & 35725.53 & 1.55 & 0.03 & 1.42 & 0.02 \\
36068.50 & 36407.98 & 1.42 & 0.03 & 1.38 & 0.01 \\
36783.79 & 36840.76 & 1.23 & 0.09 & 1.42 & 0.02 \\
38259.42 & 38289.56 & 1.22 & 0.06 & 1.38 & 0.02 \\
39350.47 & 39434.57 & 1.29 & 0.07 & 1.41 & 0.03 \\
39736.69 & 40153.57 & 1.27 & 0.06 & 1.38 & 0.01 \\
42274.51 & 42309.38 & 1.10 & 0.12 & 1.45 & 0.03 \\
42715.33 & 42752.27 & 1.35 & 0.21 & 1.44 & 0.05 \\
43392.33 & 43401.41 & 1.00 & 0.10 & 1.39 & 0.03 \\
43756.44 & 44168.38 & 1.19 & 0.11 & 1.42 & 0.02 \\
\hline & & & & &
\end{tabular}

phase of the fundamental mode (upper four panels) and the first overtone (bottom four panels) can be seen. The data are divided before and after the break. Both the starting and new values are used. Since the amplitude ratio of the first overtone to the fundamental mode is high, the improvement is more remarkable in the first overtone.

\section{Discussion}

For the stars situated in the instability strip of the HR diagram the evolutionary changes lead to small increases or decreases in their pulsation period. The changes are far too slow to be perceptible on human time scales. Nevertheless, the hope still exists that the period changes that were observed might reveal the speed and direction of their evolution if the database is extensive enough.

Long-term monitoring projects have been carried out over the last century. Nowadays the length of the database is around 50-70 years for most kinds of pulsating variable stars. Besides the traditionally used $\mathrm{O}-\mathrm{C}$ technique, new approaches have recently been published (Koen 1996; Paparó et al. 1998). Period changes are widely discussed in the literature for each kind of pulsating star.

The Joint Discussion, "Stellar Evolution in Real Time: Stellar Evolution on Human Time Scales" (Guinan \& Koch 1999) nicely summarized the present state of our knowledge from both observational and theoretical sides, in other words, the contradictory results from observation compared with the theory. 

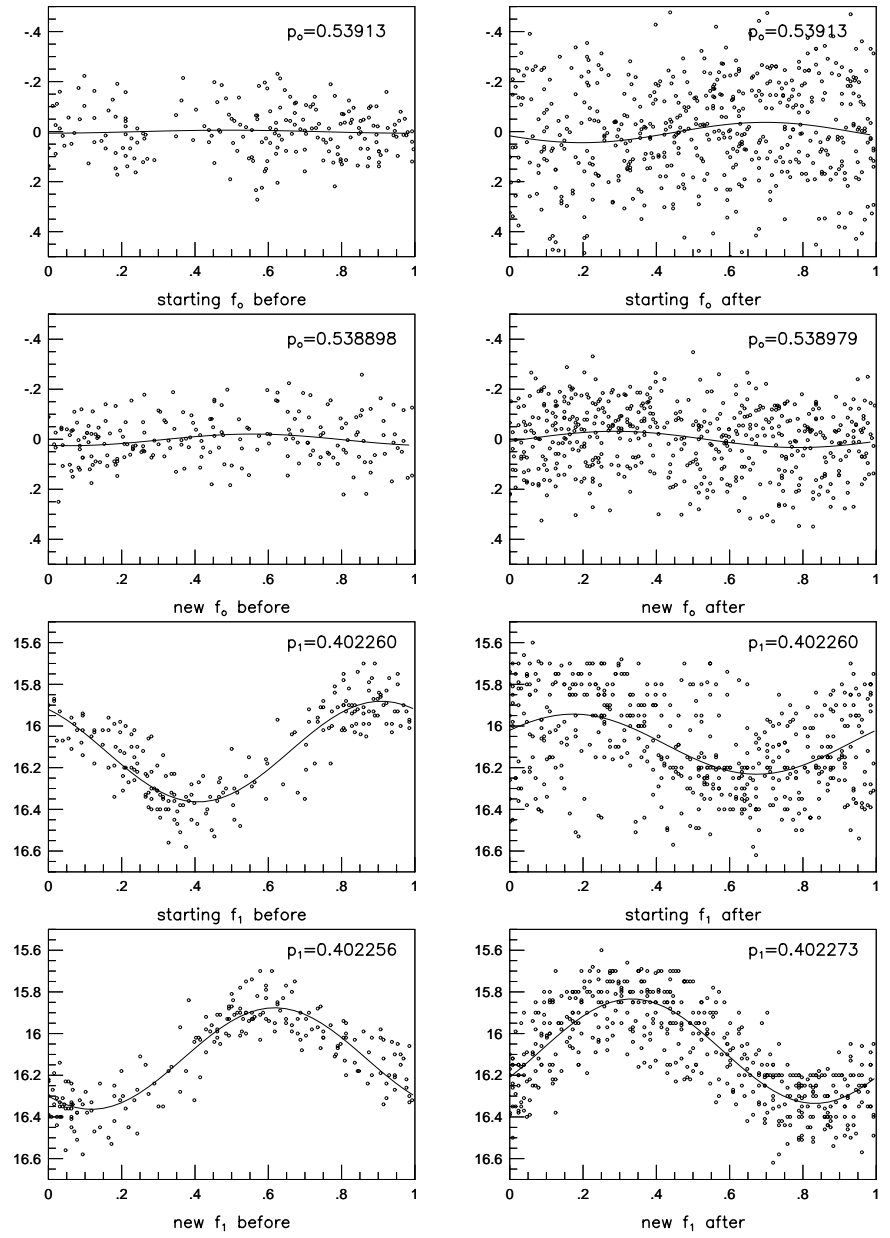

Fig. 4. Folded light curves of V26 according to $f_{0}$ and $f_{1}$, before and after the break, for the starting and new values.

All types of period change, period decrease, increase or constant period may occur in each type of pulsating variable. Some of the Cepheids show practically no period change, though most of them show a period change in agreement with the theoretical ones (Percy 1999; Szabados, private communication). The fairly good agreement for the Cepheids is a consequence of crossing the instability strip five times with a different period change rate each time. It is easy to find an evolutionary stage with period change sign and rate obtained by observation. According to the investigation of Breger (1999) equal numbers of stars with small positive and negative changes in period have been found for Pop I $\delta$ Scuti stars. The period changes of many Pop II $\delta$ Scuti (SX Phe) stars are characterized by a sudden jump (Breger $\&$ Pamyatnykh 1998). The actual situation for $\beta$ Cep stars is more complicated than is expected from theory (Jerzykiewicz 1990).

In the most remarkable cases period changes of more than one mode have been investigated and have shown period changes of different sign for simultaneously excited modes (AI Vel: Walraven et al. 1992 ( $\delta$ Scuti type), $\beta$ CMa: Shobbrook 1973 and 12 (DD) Lacertae: Pigulski 1994 ( $\beta$ Cephei type) and v53/M15: Paparó et al. 1998 (RR Lyrae type)).

It can be concluded that the observed period changes are not in agreement with those expected from a smooth stellar evolution, and we strongly suspect that the period changes are dominated by short term changes governed by the physics of the star.

The investigation of the evolution-pulsation link is still in its infancy. Period changes are estimated by the instant linear periods of static models representing different stages of evolution. This approximation can be valid for smooth and slow evolutionary changes, however for abrupt and transient-like variations, a dynamic approach is needed.

It was shown very recently that pulsation and evolution can be modelled together with the help of amplitude equations (Kolláth \& Buchler 2001; Buchler \& Kolláth 2001). From a finite number of nonlinear pulsation calculations the pulsation behaviour can be mapped on the Hertzsprung-Russell diagram, and then the period changes can be calculated for any evolutionary path. This formalism, together with slow and regular evolutionary sequences, cannot produce sudden changes (or perhaps only at bifurcations like mode switching). Sudden changes in the stellar structure are necessary to induce fast changes in the oscillation frequency, as in V26/M15. Only a couple of processes are known that can induce changes in the stellar envelope structure on a short time scale.

Sweigart \& Renzini (1979) proposed that mixing events in a small part of the semiconvective zone can produce the observed period changes. Mixing events (labelled type $B$ in Sweigart and Renzini's paper) confined to the semiconvective zone cause an increase in the period. A sudden increase in the period (or decrease in the frequency) is observable if some mixing events of that type have taken place within a short time interval (within some years). Since the periods of both modes in V26/M15 changed in a similar way, the ideas of Sweigart \& Renzini seem to have observational support. As $\Delta P / P$ is on the order of $10^{-5}$ for both modes, mixing elements amounting to $\Delta M_{\mathrm{He}} \approx 10^{-4} M_{0}$ can produce the observed period change.

Another possible mechanism for changes in the stellar envelope is the dredge up of a small amount of helium by occasional convection overshooting (Cox 1998). Since this event occurs on a short time scale, i.e. the convection turnover time, some composition inhomogeneity should be a prerequisite for this type of structure change. From the values of Table I of Cox (1998), one can estimate the necessary change in the surface helium content to be $\approx 10^{-4}$ to match the observed period changes. Whether the accretion of interstellar matter has a noticeable effect on surface composition is still an open question.

The main difference between the Sweigart \& Renzini type of structure changes and that of Cox is their location. While the dredge up on the surface can be characterized by one time scale given by the convection, mixing in the core induces changes in the star on multiple levels. After a mixing event in the semiconvective zone the radius of the star varies on the dynamic time scale, i.e. the order of the pulsation period. However, the long scale readjustment of the envelope occurs on the thermal time scale, which results in long-term period changes. For double mode stars the interaction of modes may provide an additional time scale. These effects together make the interpretation of period changes a nontrivial problem (Szabó et al. 2001).

From the theoretical point of view the period ratio of the fundamental and first overtone is a critical point for mass 
determination. It is worthwhile to check whether the abrupt period change of V26 modified the period ratio of the excited modes. Judging from the new period values the period ratio slightly decreased after the abrupt change in the periods. Before the event it was $P_{1} / P_{0}=0.74644 \pm 0.00008$ and after it $P_{1} / P_{0}=0.746361 \pm 0.000005$. If this effect were real, it would slightly modify our view about the mass - period ratio relation. The errors in determining the period ratios before and after the abrupt period changes, however, do not preclude the constancy of the period ratio.

Recent studies (Kolláth \& Buchler 2001) indicate that the difference between the nonlinear and linear period ratios of RR Lyrae models is of the order of $0.1 \%$. The actual value depends on the amplitudes, and for typical RR Lyrae models less than $10 \%$ change in the amplitudes results in a $10^{-4}$ shift in $P_{1} / P_{0}$. If the observed $\Delta\left(P_{1} / P_{0}\right) \approx 10^{-4}$ shift is real and it is mostly induced by amplitude changes due to the evolution of the star, then the amount of amplitude changes should be a few percent. Such a small amplitude variation cannot be detected in the observations with any degree of significance. The Fourier phase method is not sensitive to such a small amplitude variation.

Thorough investigations of period changes of double mode RR Lyrae stars in other globular clusters by the Fourier phase method are strongly suggested. With more, well-studied cases, there is a greater chance of distinguishing between the period changes caused by occasional changes in the physics of the star and the evolutionary changes.

Acknowledgements. Research grants, OTKA Nos. T 030955, $\mathrm{T} 038440$ and $\mathrm{T} 025288$ are gratefully acknowledged. The authors are indebted to Dr. N. A. Silbermann for her very thorough referee report and helpful comments.

\section{References}

Bailey, S. I. 1919, Harvard Ann., 78, 195

Barlai, K. 1989, Commun. Konkoly Obs., 10, No. 92, 143

Bingham, E. A., Cacciari, C., Dickens, R. J., \& Fussi Pecci, F. 1984, MNRAS, 209, 765

Breger, M. 1999, New Astron. Rev., 43, 441

Breger, M., \& Pamyatnykh, A. A. 1998, A\&A, 332, 958

Buchler, J. R., \& Kolláth, Z. 2001, ApJ, submitted

Clement, C. M., \& Walker, I. R. 1991, AJ, 101, 1352

Cox, A. N. 1998, ApJ, 496, 246
Cox, A. N., Hodson, S. W., \& Clancy, S. P. 1983, ApJ, 266, 94

Filippenko, A. V., \& Simon, R. S. 1981, AJ, 86, 671

Fritze, K. 1963, Astron. Nachr., 287, 79

Gordenko, A. F., Klabukova, A. V., \& Fashchevskij, N. N. 1984, Prob. Kosmicheskoj Fiz., 19, 93

Grubissich, C. 1956, Mem. Soc. Astr. It., 27, 391

Guinan, E. F., \& Koch, R. H. 1999, New Astron. Rev., 43, vii

Hodson, S. W., \& Cox, A. N. 1982, in Pulsation in Classical and Cataclysmic Variable Stars, ed. J. P. Cox, \& C. J. Hansen (JILA, Boulder), 201

Hoffmeister, C., Richter, G., \& Wenzel, W. 1985, Variable Stars (Springer-Verlag, Berlin, Heidelberg, New York, Tokyo)

Jerzykiewicz, M. 1999, New Astron. Rev., 43, 455

Jurcsik, J., \& Barlai, K. 1990, in Confrontation Between Stellar Pulsation and Evolution, ed. C. Cacciari, \& G. Clementini, 112

Koen, C. 1996, MNRAS, 283, 471

Kolláth, Z., \& Buchler, J. R. 2001, in Stellar Pulsation - Nonlinear Studies, ed. M. Takeuti, \& D. D. Sasselov, ASSL, 257, 29

Kovács, G., Shlosman, I., \& Buchler, J. R. 1986, ApJ, 307, 593

Makarova, V. A., \& Akimova, V. P. 1965, Perem. Zvezdy, 15, 350

Nemec, J. M. 1985, AJ, 90, 240

Paparó, M., Saad, S. M., Szeidl, B., et al. 1998, A\&A, 332, 102 (Paper I)

Paparó, M., Saad, S. M., Szeidl, B., Kolláth, Z., \& Abu Elazm 1997, in Sounding Solar and Stellar Interiors, Poster papers, ed. J. Provost, \& F.-X. Schmider, IAU Symp., 181, 275

Percy, J. R. 1999, New Astron. Rev., 43, 449

Pigulski, A. 1994, A\&A, 292, 183

Purdue, P., Silbermann, N. A., Gay, P., \& Smith, H. A. 1995, AJ, 110, 1712

Sandage, A., Katem, B., \& Sandage, M. 1981, ApJS, 46, 41

Sawyer Hogg, H. 1973, Publ. David Dunlap Obs., 3, No. 6

Silbermann, N. A. 2001, private communication

Silbermann, N. A., \& Smith, H. A. 1995a, AJ, 109, 1119

Silbermann, N. A., \& Smith, H. A. 1995b, AJ, 110, 704

Smith, H. A., \& Sandage, A. 1981, AJ, 86, 1870

Shobbrook, R. R. 1973, MNRAS, 161, 257

Stothers, R. 1980, PASP, 92, 475

Sweigart, A. V., \& Renzini, A. 1979, A\&A, 71, 66

Szabados, L. 2000, private communication

Szabó, R., Kolláth, Z., Csubry, Z., \& Buchler, J. R. 2001, in Astrophysical Ages and Time scales, ed. T. von Hippel, N. Manset, \& C. Simpson, ASP Conf. Ser., 245, 373

Szeidl, B. 1975, in Variable Stars and Stellar Evolution, ed. V. E. Sherwood, \& L. Plaut (Reidel, Dordrecht) IAU Symp., 67, 545

Walraven, Th., Walraven, J., \& Balona, L. A. 1992, MNRAS, 254, 59 Wesselink, A. J. 1974, A\&A, 36, 163 